\title{
Robust Content-Based Digital Image Watermarking Scheme in Steerable Pyramid Domain
}

\author{
Khadija Jamali ${ }^{1,2}$, Mohamed El Aroussi ${ }^{2}$, Azz El Arab El Hossaini ${ }^{1,2}$, Samir Mbarki ${ }^{1}$ \& Mohammed Wahbi $^{2}$ \\ ${ }^{1}$ Departement of Computer Science, Faculty of Science, Ibn Tofail University, Kenitra, Morocco \\ ${ }^{2}$ Department of Electrical Engineering, Hassania School of Public Works, Casablanca, Morocco \\ Correspondence: Khadija Jamali, Departement of Computer Science, Faculty of Science, Ibn Tofail University, \\ Kenitra, Morocco. Tel: 212-537-32-94-00. E-mail: jamalikhadija@gmail.com
}

Received: December 11, 2015

doi:10.5539/cis.v9n2p99
Accepted: December 30, 2015 Online Published: April 19, 2016

URL: http://dx.doi.org/10.5539/cis.v9n2p99

\begin{abstract}
In the digital world in which we are living, the intellectual property protection becomes a concern especially with the proliferation of files transfer over networks. The ability to access data such as text, images, video, and audio has become quicker and easier for people with little to no knowledge of technology. In this paper, a robust watermarking scheme based on the original image content is proposed and simulated. Steerable pyramid transform is used as an embedding domain to its good spatial-frequency characteristics, and its wide applications in the image/video coding standards. The embedding process aims to insert some information in a digital document to identify its owner later. This process requires the original image to be protected and the watermark image related to the image's owner. It needs also a threshold value used by Sobel-Feldman operator to extract the original image features. The embedding of the watermark image is performed in high frequency components of the original image. Experimental evaluation demonstrates that the proposed watermarking scheme is able to withstand a variety of attacks and at the same time provide good visual quality of the watermarked image.
\end{abstract}

Keywords: watermarking, robustness, steerable pyramid transform, geometric attacks

\section{Introduction}

With the extreme development of network technology and internet, the protection of intellectual property and authentication become very important. To secure and limit the illegal distribution of digital images, many solutions are proposed in the literature. Among these solutions, only watermarking technologies can protect the digital content after been received by a user.

Digital watermarking is an advantageous solution to aforementioned issues (Cheddad, A., Condell, J., Curran, K., \& Mc Kevitt, P, 2010). Image watermarking technologies consist on hiding secret information in an image called original image or host image. The inserted information is called watermark, and the image where the watermark is inserted, is called watermarked image or protected image. Thereafter, the watermark could be extracted or detected in order to use it if necessary to verify ownership and/or to distinguish product user.

According to human perception, watermarking schemes can be classified as visible or invisible watermarking. A Visible watermarking scheme means that the watermark can be seen on the protected image by human perception. For a better protection, the inserted watermark should be invisible. Indeed, a visible watermark can easily be modified or erased by another watermark. Thereby, ensuring the invisibility of the watermark returns to respect the visual quality of the proposed product. For this reason, several existing watermarking schemes as the proposed watermarking scheme in this paper tend to perform insertion into the less sensitive areas of interest to the human perception.

Digital image watermarking is performed in spatial or frequency domains. Spatial domain schemes are based on direct manipulation of pixels to embed a watermark. Such schemes are simple and fast to implement, although they are not robust against the majority of attacks. Compared to the spatial domain, transform domain schemes, for instance discrete wavelet transforms (DWT), discrete cosine transforms (DCT), and Discrete Fourier Transform (DFT), and Fourier-Mellin transform, singular value decomposition (SVD), are more robust against attacks. This is due to the fact that the image is transformed to its frequency representation to embed the watermark. 
Several watermarking schemes using different concepts are proposed in the literature. Authors in (W. Lin, S. Horng, T. Kao, P. Fan, C. Lee, Y. Pan, 2008), Propose a scheme based on significant difference of wavelet coefficient quantization. DWT has also attracted interest, (B. Ma, Y. Wang, C. Li, Z. Zhang, D. Huang, 2012) designed a robust watermarking method where the watermark is distributed on the maximum positive and minimum negative coefficients. (S. Wang, Y. Lin, 2004) proposed a wavelet-tree-based watermarking scheme; the wavelet coefficients are grouped into so-called super trees. The insertion is done by quantizing super trees. (W. Lin, Y. Wang, S. Horng, T. Kao, Y. Pan, 2009) enhances the robustness of the scheme by inserting the watermark in the local maximum coefficient. (Li, C., Zhang, Z., Wang, Y., Ma, B., \& Huang, D., 2015) designed a scheme that represents a dither modulation of significant amplitude difference for wavelet based robust watermarking. Differently from the above schemes, (Yahya, A. N., Jalab, H. A., Wahid, A., \& Noor, R. M., 2015) use discrete wavelet and probabilistic neural network to guarantee the robustness while preserving good impeccability. By combining Fuzzy Logic and Discrete Cosine Transform, (Jagadeesh, B., Kumar, P. R., \& Reddy, P. C, 2015) realize a robust digital image watermarking technique. (Agarwal, C., Mishra, A., \& Sharma, A, 2015) implement a robust image watermarking scheme to embed a permuted binary watermark in gray-scale images.

Based on steerable pyramid and the original image content, this paper proposes a robust watermarking scheme. Firstly steerable pyramid transform is used to decompose the original image into several subbands to choose the low subband that will carry the watermark image. Areas to be watermarked are chosen based on the image contents that are less sensitive to the human eye. The embedding of the watermark image is performed in high frequency components of the original image that are extracted by using Sobel-Feldman operator to extract. After embedding the watermark image, the inverse steerable pyramid is performed to get the watermarked image.

The rest of this paper is organized as follows. Section 2 describes the steerable pyramid transform which is a multi-scale, multi-orientation transform. Section 3 covers the details of the watermark insertion and detection scheme. Results in Section 4 will show the high performance of our scheme in terms of robustness and impermeability. Finally, Section 5 concludes the paper.

\section{Embedding Domain}

In the proposed watermarking scheme, the watermark image is inserted in multiresolution domain that represents a very interesting watermarking space. In this approach, the original image is sub-sampling recursively until reaching the desire result. The original image is filtered using a low pass filter to forming a new image whose size is four times smaller. This process attempts to mimic the natural mechanism of resolution visual loss effect.

In this paper, we use the Steerable Pyramid transform introduced by (Freeman, W. T., \& Adelson, E. H, 1991) as the embedding domain for our proposed watermarking scheme as we did for several watermarking schemes that are already published in the literature. Among them, we can cite the following works, In (Jamali, K., El Aroussi, M., El Hossaini, A. E. A., Mbarki, S., \& Wahbi, M, 2014) we presented a robust scheme using steerable pyramide, to embed the watermark, firsty the steerable pyramid transform is applied on the both of the watermark and host image. Then deferently to others scheme we choose to insert the watermark on all levels and all orientations of the pyramid. This scheme is robust against cropping attack, for the reason that the watermark is inserted several times, the watermark is detected from the remaining part. In (Hossaini, E., El Arab, A., El Aroussi, M., Jamali, K., Mbarki, S., \& Wahbi, M. (2014), we presented a new, robust digital watermarking scheme for ownership protection. In the watermarking process and after performing steerable pyramid on the original image, two independent streams representing the watermark are embedded in low and mid-frequency of DCT components. Watermark detection is based on comparison result between Pearson product moment correlations of the two independent streams with each watermarked block. In (El Hossaini, A. E. A., El Aroussi, M., Jamali, K., Mbarki, S., \& Wahbi, M, 2014), we proposed a highly robust digital image watermarking scheme based on steerable pyramid transform and dual encryption technique. Before the embedding process a pretreatment on the watermark image is performed to enhance the security using Arnold transform and a proposed encryption function. The host image is decomposed into steerable pyramid coefficients and the low-subband is selected to receive the encrypted watermark. The same Keys used during the embedding process are also used during the extraction process. Unlike traditional watermarking schemes, in (El Hossaini, A. E. A., El Aroussi, M., Jamali, K., Mbarki, S., \& Wahbi, M, 2016) we proposed a watermarking method that does not alter the original image by embedding the watermark image. Steerable pyramid transform is performed on the original image, and the low sub-band is selected. The watermark image is divided into two random looking images, called private and public shares using the visual secret sharing scheme and the selected low sub-band features. To reveal the watermark image, the two shares are stacked together. 
In(Jamali, K., El Aroussi, M., El Hossaini, A. E. A., Mbarki, S., \& Wahbi, M, 2014) we presented a robust scheme using steerable pyramide, to embed the watermark, firsty the steerable pyramid transform is applied on the both of the watermark and host image. Then deferently to others scheme we choose to insert the watermark on all levels and all orientations of the pyramid. This scheme is robust against cropping attack, for the reason that the watermark is inserted several times, the watermark is detected from the remaining portion.

The Steerable Pyramid transform is a linear multi-scale, multi-orientation image decomposition that provides a useful front-end for image-processing and computer vision applications (Freeman, W. T., \& Adelson, E. H, 1991) Unlike other existing pyramid transform as laplacian pyramid and Gaussian pyramid, the number of scales and directions is variable.

The block diagrams for analysis and synthesis decomposition are shown in Figure 1 and Figure 2. The steerable pyramid transform uses three types of filters: low-pass (L0), high-pass (H0), and pass-bands (B0... BK). By using the low-pass and high-pass filters, the image is separated into low and high-pass subbands. Then, the lowpass subband is further decomposed into a set of oriented band-pass subbands and a low-pass subband. And finaly, this low-pass subband is subsampled by a factor of 2 in the $\mathrm{X}$ and $\mathrm{Y}$ directions, and a new decomposition is performed until reaching the scale fixed by the user.

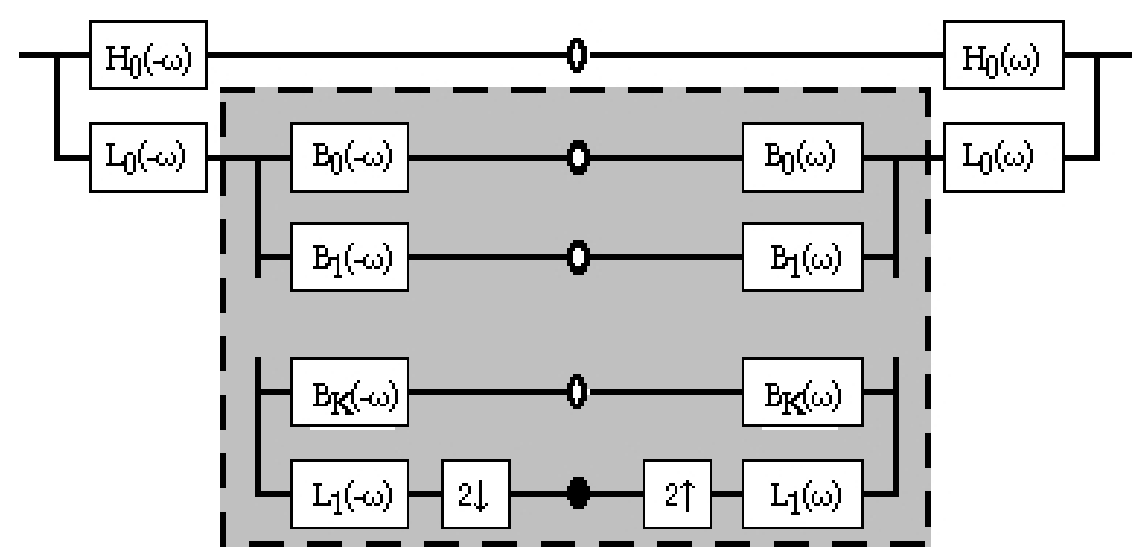

Figure 1. Tree representation of one-level 2D steerable pyramid transform

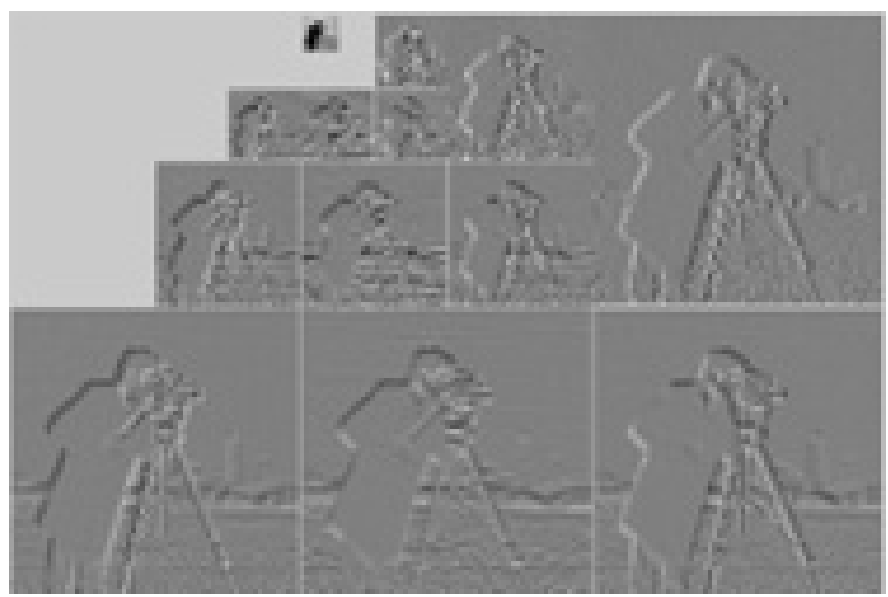

Figure 2. Tree Cameraman steerable pyramid-based image decomposition

\section{The Proposed Watermarking Scheme}

In general, a watermarking scheme is divided into two basic parts: the embedding and the detection of the watermark.

The embedding process aims to insert some information in a digital document to identify its owner later. This process requires the original image to be protected and the watermark image related to the digital document's owner. Another optional parameter called secret key may be used if necessary in some cases where the proposed 
watermarking schemes needs to scramble the watermark image to overcome some securities issues. The secret key could be used also to formatting the watermark image or to locating areas in which the embedding process should be performed (Lee, C. H., \& Lee, Y. K, 1999).

The embedding process in our proposed watermarking scheme needs the original image, the watermark image and the threshold value used by Sobel-Feldman operator to extract the original image features. The embedding of the watermark image is performed in high frequency components of the original image. These components are represented by edges which give details to images and make the watermark scheme more robust to the majority of attack because these details will remain present in the watermarked image even if several degradations occur on it. Moreover, digital image pirates will not be interested in stealing an image that has being seriously distorted (because it becomes worthless).

The proposed watermarking scheme in this article is classified as non-blind watermarking schemes. To extract the watermark image previously inserted in the original image, the watermark extraction process needs the original image, the watermarked image and the secret key represented by the threshold value used by Sobel-Feldman operator.

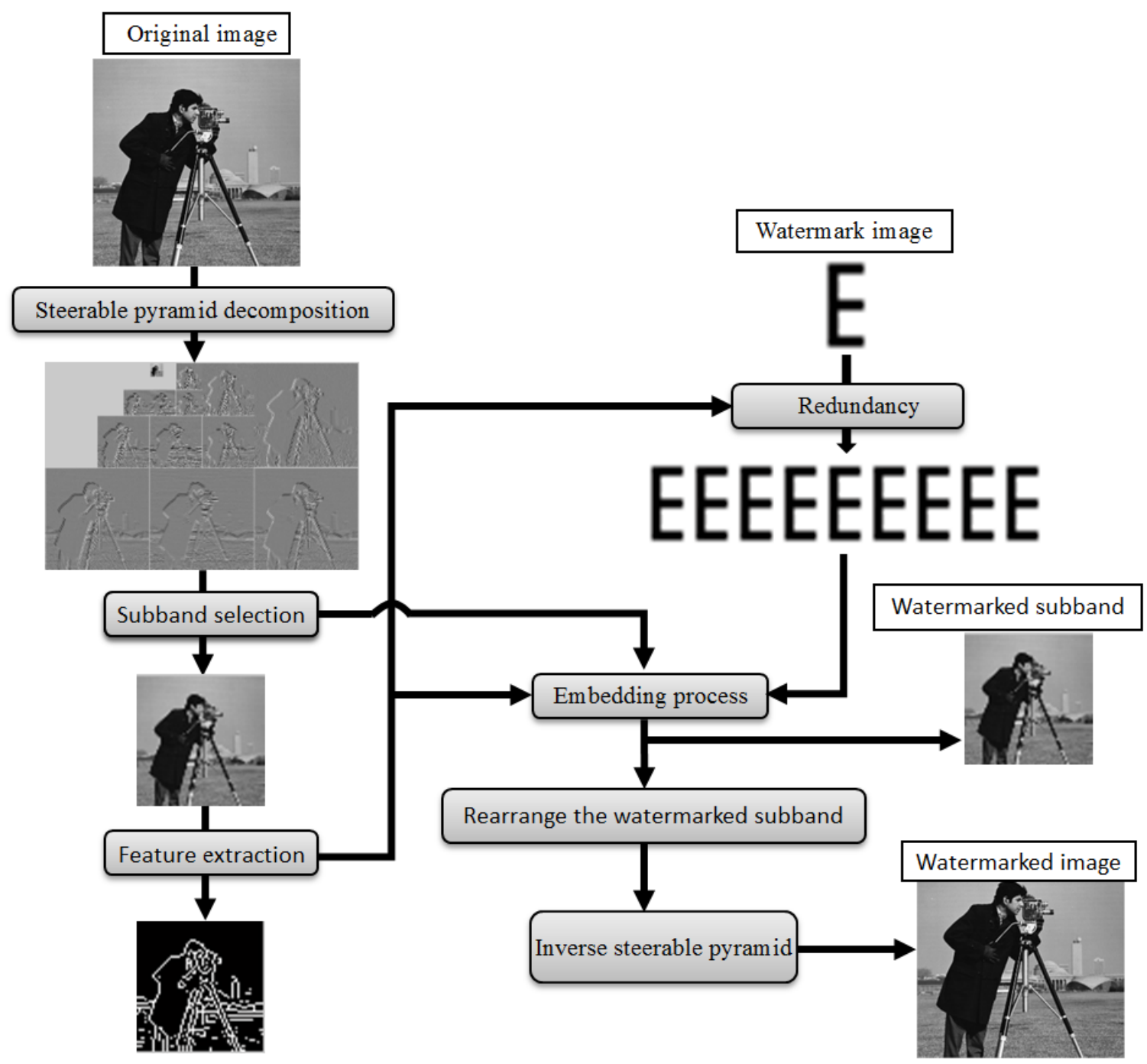

Figure 3. Block diagram of the proposed watermark embedding scheme

\subsection{Watermark Embedding Process}

The process of watermark embedding is shown in Figure 3, and the detailed algorithm is given as follows:

Step 1) The low subband Lsb is selected after decomposing the original image Oi into several subbands using one scale and two orientations steerable pyramid transform. 
Step 2) Based on threshold value T1, the low subband features Lsf are extracted by performing Sobel-Feldman operator.

$$
L_{s f}=\text { sobel_edge_detection }\left(O_{i}, T_{1}\right)
$$

Step 3) The watermark image can be inserted several times in the original image if its size is less than the total number of extracted edge pixels. The watermark image Wo is formatted to a new Watermark image Wr using a redundancy process that duplicate the original image $\mathrm{R}$ times.

$$
\mathrm{R}=\frac{\text { total number of extracted edge pixels }}{\text { total number of watermark image pixels }}
$$

Where $\mathbf{R}$ is the number of watermark redundancy.

Step 4) for each edge pizels location in Lsf the equation 3 is used to embed the formatted watermark's bit of value 1 and equation 4 is used to embed the formatted watermark's bit of value 0 .

$$
\begin{gathered}
L_{s b w}(i, j)=L_{s b}(i, j)-\text { alpha } \\
L_{s b w}(i, j)=L_{s b}(i, j)+\text { alpha }
\end{gathered}
$$

alpha : is the watermark strength that must be adjusted to compromise between robustness and visibility of the watermark.

$\mathbf{L}_{\mathbf{s b w}}(\mathbf{i}, \mathbf{j}) \quad$ : represent a pixel value in the watermarked low subband.

$\mathbf{L}_{\mathbf{s b}}(\mathbf{i}, \mathbf{j}) \quad$ : represent a pixel value in the original low subband.

$\mathbf{i}, \mathbf{j} \quad$ : represent edge pizels location in Lsf.

Step 5) After rearranging each subband to its initial location, the watermarked image is obtained by performing the inverse steerable pyramid transform.

\subsection{Watermark Extraction Process}

The process of watermark extraction is shown in Figure 4, and the detailed algorithm is given as follows:

Step 1) The low subband $\boldsymbol{L}_{\boldsymbol{s} b}$ is selected after decomposing the original image $\boldsymbol{O}_{\boldsymbol{i}}$ into several subbands using one scale and two orientations steerable pyramid transform.

Step 2) The same as the step 2 in the embedding process, the low subband features $\boldsymbol{L}_{\boldsymbol{s} f}$ are extracted by performing Sobel-Feldman operator.

Step 3) the same as step 3 in the embedding process, calculate the number $\boldsymbol{R}$ that represent the number of watermark redundancy.

Step 4) Construct an empty matrix $\boldsymbol{W}_{\boldsymbol{f}}$ of size $\boldsymbol{R}$ times the size of the original watermark.

Step 5) The low subband $\boldsymbol{L}_{\boldsymbol{w s b}}$ is selected after decomposing the watermarked image $\boldsymbol{O}_{\boldsymbol{w}}$ into several subbands using one scale and two orientations steerable pyramid transform.

Step 6) for each edge pixels location in $\boldsymbol{L}_{s f}$, the formatted watermark $\boldsymbol{W}_{\boldsymbol{f e}}$ is extracted as follow:

$$
W_{f e}=\left\{\begin{array}{lll}
0, & \text { if } & \left(L_{w s b}-L_{s b}\right)<0 \\
1, & \text { if } & \left(L_{w s b}-L_{s b}\right)>=0
\end{array}\right.
$$

Step 7) Divide the extracted $\boldsymbol{W}_{\boldsymbol{f}}$ image into $\boldsymbol{R}$ blocks of the same size as the original watermark. Then calculate the average of the $\boldsymbol{R}$ blocks and perform a threshold to make a decision of each watermark's bit.

$$
W_{e}(a, b)=\left\{\begin{array}{l}
1, \quad \text { if } \operatorname{avg}\left(\sum_{k=1}^{R} B_{\text {lock }}(a, b)\right)>\text { Threshold } \\
0, \quad \text { if } \operatorname{avg}\left(\sum_{k=1}^{R} B \operatorname{lock} k_{k}(a, b)\right)<=\text { Threshold }
\end{array}\right.
$$

Where $\boldsymbol{W}_{e}$ is the extracted watermark, and $(\boldsymbol{a}, \boldsymbol{b})$ is the location of one pixel. 


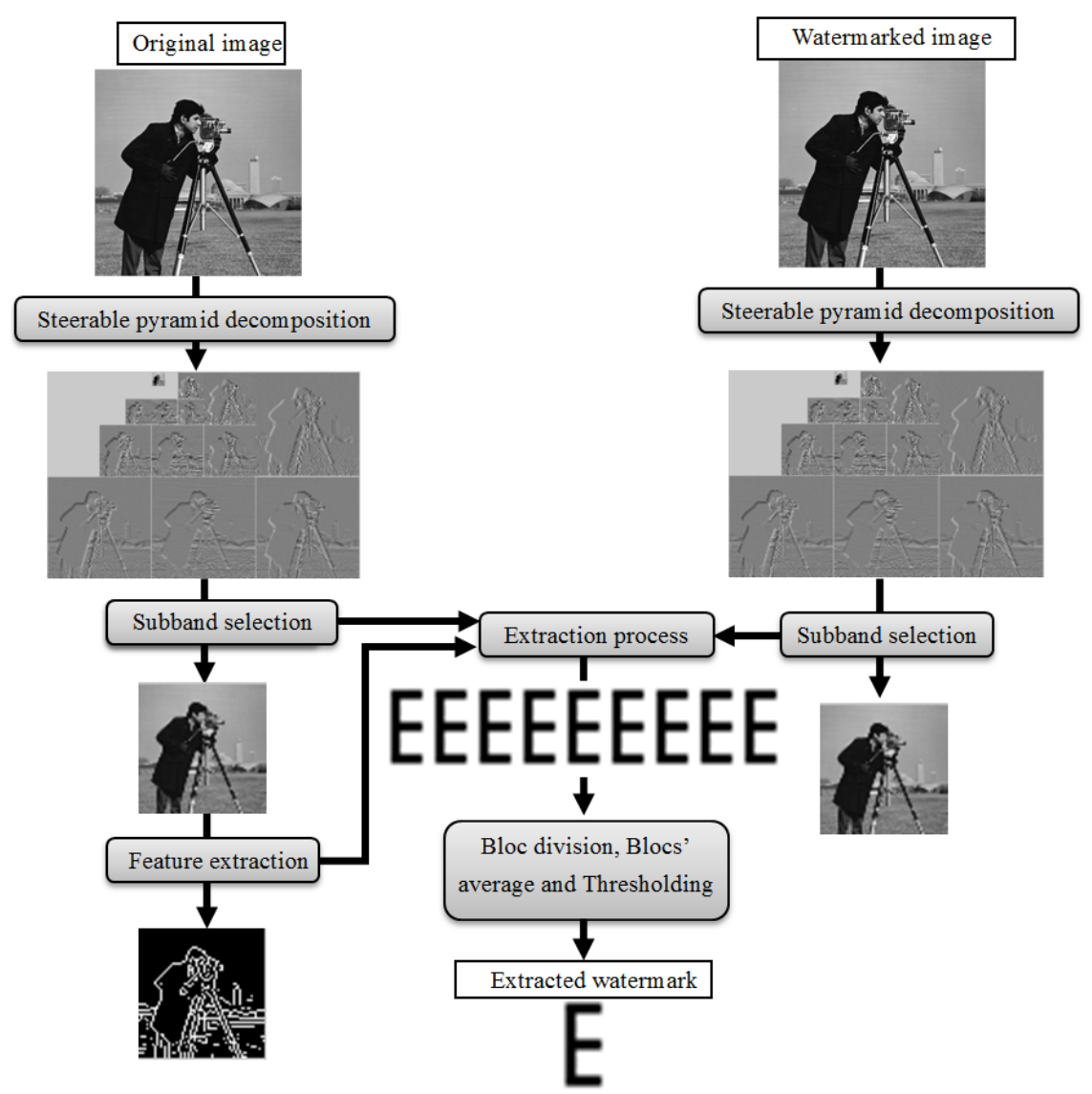

Figure 4. Block diagram of the proposed watermark extraction scheme

\section{Experimental Results}

In this section we present the experimental results of the proposed watermarking scheme and we compare its performance with the results reported in other existing watermarking schemes.

To get optimal results, some tests are performed to choose optimal configuration settings for the proposed watermarking scheme. Table 1 and Table 2 present various results based on the quality of the obtained watermarked images and the extracted watermarks. These results are obtained by testing different number of filters and scales using the Einstein test image for Table 1 and the Cameraman test image for Table 2. From results in Table 1 and Table 2 we can see that our algorithm obtains better performance when using: one scale / two filters, two scales / one filter and two scales / two filters.

Table 1. NC and PSNR values using different number of scales and filters for Einstein and Cameraman image.

\begin{tabular}{|c|c|c|c|c|c|c|c|}
\hline \multicolumn{4}{|c|}{ Einstein image } & \multicolumn{4}{|c|}{ Cameraman image } \\
\hline $\begin{array}{l}\text { Number of } \\
\text { scales }\end{array}$ & $\begin{array}{l}\text { number of } \\
\text { filter }\end{array}$ & $P S N R(d B)$ & $N C$ & $\begin{array}{l}\text { Number } \\
\text { of scales }\end{array}$ & $\begin{array}{l}\text { number of } \\
\text { filter }\end{array}$ & $P S N R(d B)$ & $N C$ \\
\hline 1 & 1 & 51.2895 & 0.9756 & 1 & 1 & 46.9305 & 1 \\
\hline 1 & 2 & 48.0474 & 1 & 1 & 2 & 44.8357 & 1 \\
\hline 1 & 4 & 48.1691 & 0.9969 & 1 & 4 & 45.0036 & 1 \\
\hline 1 & 6 & 47.2767 & 0.9937 & 1 & 6 & 44.3003 & 1 \\
\hline 2 & 1 & 49.4362 & 1 & 2 & 1 & 50.0355 & 0.9937 \\
\hline 2 & 2 & 46.0881 & 1 & 2 & 2 & 46.0196 & 1 \\
\hline 2 & 4 & 46.0676 & 1 & 2 & 4 & 45.9653 & 1 \\
\hline 2 & 6 & 44.5125 & 0.9969 & 2 & 6 & 43.2525 & 0.9969 \\
\hline 3 & 1 & 51.3001 & 0.9969 & 3 & 1 & 52.9769 & 0.9713 \\
\hline
\end{tabular}




\begin{tabular}{llllllll}
3 & 2 & 45.9120 & 0.9969 & 3 & 2 & 45.0242 & 0.9841 \\
3 & 4 & 45.7946 & 0.9969 & 3 & 4 & 44.7877 & 0.9713 \\
3 & 6 & 43.2829 & 0.9969 & 3 & 6 & 41.0016 & 0.8751 \\
4 & 1 & 54.9405 & 0.9727 & 4 & 1 & 54.5913 & 0.9152 \\
4 & 2 & 45.0246 & 0.9557 & 4 & 2 & 43.5148 & 0.9129 \\
4 & 4 & 44.7729 & 0.9464 & 4 & 4 & 43.0730 & 0.9193 \\
4 & 6 & 41.8308 & 0.8599 & 4 & 6 & 39.8229 & 0.7690 \\
5 & 1 & 57.5026 & 0.5396 & 5 & 1 & 56.4748 & 0.4990 \\
5 & 2 & 39.2322 & 0.4593 & 5 & 2 & 40.0926 & 0.4224 \\
5 & 4 & 39.1915 & 0.4593 & 5 & 4 & 39.7250 & 0.4246 \\
5 & 6 & 38.0444 & 0.3899 & 5 & 6 & 37.0135 & 0.3914 \\
\hline
\end{tabular}

Table 2. NC and PSNR values using different number of scales and filters for different images.

\begin{tabular}{lllllll}
\hline & one scale and two filters & \multicolumn{3}{c}{ two scales and one filter } & \multicolumn{2}{c}{ two scales and two filters } \\
\cline { 2 - 7 } Images & $P S N R$ & $N C$ & $P S N R$ & $N C$ & $P S N R$ & $N C$ \\
\hline Goldhill & 44.4756 & 1 & 47.1695 & 1 & 43.1861 & 1 \\
Lena & 45.3607 & 1 & 48.3115 & 1 & 45.0064 & 1 \\
Peppers & 40.3727 & 1 & 47.5341 & 0.9876 & 37.9530 & 1 \\
Barbara & 43.7066 & 1 & 47.2353 & 1 & 42.7938 & 1 \\
Baboon & 39.7874 & 1 & 47.6290 & 0.9969 & 35.6088 & 1 \\
Einstein & 48.0474 & 1 & 49.4362 & 1 & 46.0881 & 1 \\
Cameraman & 44.8357 & 1 & 50.0355 & 0.9937 & 46.0196 & 1 \\
\hline
\end{tabular}

To make a decision about the number of scales and filters to use by our watermarking scheme, we performed more tests using other popular images (Goldhill, Lena, Peppers, Barbara and Baboon). These tests and its results are shown in Table 3 in which we used various parameters (one scale / two filters, two scales / one filter and two scales / two filters). By considering the quality of the obtained watermarked images and the extracted watermarks, the following parameters are selected for the steerable pyramid transform: number of filters $=2$ and number of scales $=1$.

\subsection{Imperceptibility of the wWatermark}

To evaluate the impact of the embedding process on the watermarked image in terms of imperceptibility, we applied our proposed watermarking scheme on seven grayscale images (Goldhill, Lena, Peppers, Barbara, Baboon, Einstein and Cameraman) using a binary watermark image represented by the letter "E".

Table 3 displays the host image, the watermark image, the watermarked image and the extracted watermark. . In our experiments, The PSNR (Peak Signal to Noise Ratio) values obtained are above $46 \mathrm{~dB}$ for all test images which demonstrates a high imperceptibility of the watermark method.

$$
P S N R=10 \log _{10} \frac{255^{2}}{M S E}
$$

Where MSE (Mean Square Error) is defined as:

$$
\operatorname{MSE}=\frac{1}{N} \sum_{i=1}^{N}\left(I_{i}-?_{i}^{2}\right.
$$

Where $\mathrm{N}$ represents the number of pixels in the original $(I)$ and watermarked $(\hat{I})$ image.

Table 3. The original image, the watermark image, the watermarked image, and the extracted watermark using

\begin{tabular}{|c|c|c|c|c|}
\hline Original Image & $\begin{array}{c}\text { Watermark } \\
\text { image }\end{array}$ & $\begin{array}{c}\text { Watermarked } \\
\text { image }\end{array}$ & $\begin{array}{l}\text { Extracted } \\
\text { watermark }\end{array}$ & $P S N R$ \\
\hline
\end{tabular}
our scheme 


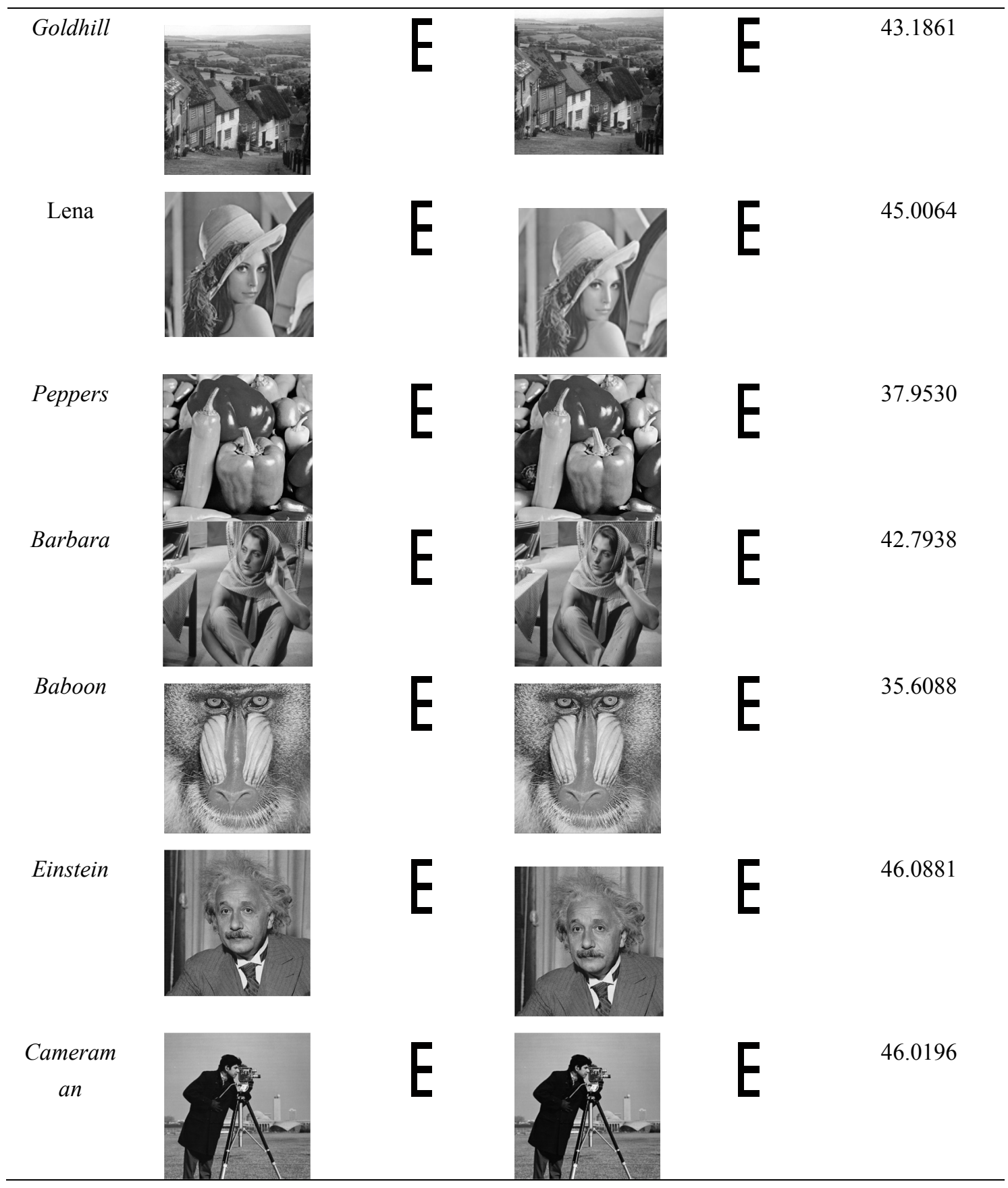

\subsection{Robustness}

The robustness of the proposed technique is validated against several attacks like JPEG compression, rotation, Gaussian noise, Speckle noise, Salt and pepper noise, Gaussian filter, Median filter, Average filter, Sharpening and Cropping. The steerable pyramid parameters used in our experiments are: number of scales $=1$ and number of orientations $=2$. The test images used are: Lena, Cameraman, Einstein and Zelda. In this work, we used the normalized correlation (NC) to measure the similarity between the original $W$ and the extracted watermark $\widehat{W}$.

$$
\mathrm{N} \mathrm{C}=\frac{\sum_{i=1}^{M} W_{i} \widehat{W}_{i}}{\sqrt{\sum_{i=1}^{M} W_{i}^{2}} \sqrt{\sum_{i=1}^{M} \widehat{W}_{i}^{2}}}
$$

The Bit error rate (BER) is used too as the $\mathrm{NC}$ to evaluate the robustness of watermark. 


$$
B E R=\frac{\sum_{i=1}^{N} \widehat{W}_{i} \oplus W_{i}}{M}
$$

Where $\mathrm{M}$ is the total size of the watermark and $\oplus$ is the xor operator.

The NC values of the extracted watermarks are very close or equal to 1 for the majority of results and the BER values are close or equal to 0 . These results demonstrate that our scheme is robust against all attacks used in these experiments.

Table 4. NC and BER values of the extracted watermark under different attacks

\begin{tabular}{|c|c|c|c|}
\hline Attack & Image & $\mathrm{NC}$ & BER \\
\hline \multirow{4}{*}{$\operatorname{JPEG}(\mathrm{Q}=90)$} & Lena & 1 & 0 \\
\hline & Cameraman & 1 & 0 \\
\hline & Einstein & 1 & 0 \\
\hline & Zelda & 1 & 0 \\
\hline \multirow[t]{4}{*}{$\mathrm{JPEG}(\mathrm{Q}=75)$} & Lena & 1 & 0 \\
\hline & Cameraman & 1 & 0 \\
\hline & Einstein & 1 & 0 \\
\hline & Zelda & 1 & 0 \\
\hline \multirow[t]{4}{*}{$\mathrm{JPEG}(\mathrm{Q}=50)$} & Lena & 1 & 0 \\
\hline & Cameraman & 1 & 0 \\
\hline & Einstein & 1 & 0 \\
\hline & Zelda & 1 & 0 \\
\hline \multirow[t]{4}{*}{ JPEG $(Q=25)$} & Lena & 1 & \\
\hline & Cameraman & 0.9937 & 0.0091 \\
\hline & Einstein & 1 & 0 \\
\hline & Zelda & 0.9969 & 0.0045 \\
\hline \multirow[t]{4}{*}{$\mathrm{JPEG}(\mathrm{Q}=10)$} & Lena & 0.9571 & 0.0636 \\
\hline & Cameraman & 0.9485 & 0.0773 \\
\hline & Einstein & 1 & 0 \\
\hline & Zelda & 0.9665 & 0.0500 \\
\hline \multirow{4}{*}{ Rotation $\left(180^{\circ}\right)$} & Lena & 1 & 0 \\
\hline & Cameraman & 1 & 0 \\
\hline & Einstein & 1 & 0 \\
\hline & Zelda & 1 & 0 \\
\hline \multirow{4}{*}{$1 \%$ Gaussian noise } & Lena & 0.9330 & 0.1000 \\
\hline & Cameraman & 0.8917 & 0.1545 \\
\hline & Einstein & 0.9132 & 0.1364 \\
\hline & Zelda & 0.9485 & 0.0773 \\
\hline \multirow{4}{*}{ Speckle noise 0.01} & Lena & 0.9845 & 0.0227 \\
\hline & Cameraman & 0.9845 & 0.0227 \\
\hline & Einstein & 0.9937 & 0.0091 \\
\hline & Zelda & 0.9235 & 0.1182 \\
\hline \multirow{4}{*}{ Salt \& pepper noise (density 0.03 ) } & Lena & 0.9542 & 0.0682 \\
\hline & Cameraman & 0.9534 & 0.0682 \\
\hline & Einstein & 0.9538 & 0.0682 \\
\hline & Zelda & 0.9401 & 0.0864 \\
\hline \multirow{4}{*}{ Gaussian filter } & Lena & 0.9906 & 0.0136 \\
\hline & Cameraman & 0.9167 & 0.1273 \\
\hline & Einstein & 0.9641 & 0.0545 \\
\hline & Zelda & 0.9815 & 0.0273 \\
\hline \multirow{3}{*}{ Median filter (aperture $=3.0$ ) } & Lena & 1 & 0 \\
\hline & Cameraman & 0.9969 & 0.0045 \\
\hline & Einstein & 0.9937 & 0.0091 \\
\hline
\end{tabular}




\begin{tabular}{|c|c|c|c|}
\hline & Zelda & 1 & 0 \\
\hline \multirow{4}{*}{ Median filter $($ aperture $=5.0$ ) } & Lena & 0.9906 & 0.0136 \\
\hline & Cameraman & 0.9448 & 0.0864 \\
\hline & Einstein & 0.9845 & 0.0227 \\
\hline & Zelda & 0.9727 & 0.0409 \\
\hline \multirow{4}{*}{ Weiner filter (aperture $=3.0$ ) } & Lena & 1 & 0 \\
\hline & Cameraman & 1 & 0 \\
\hline & Einstein & 1 & 0 \\
\hline & Zelda & 1 & 0 \\
\hline \multirow{4}{*}{ Average filter $3 \times 3$} & Lena & 1 & 0 \\
\hline & Cameraman & 0.9937 & 0.0091 \\
\hline & Einstein & 0.9969 & 0.0045 \\
\hline & Zelda & 0.9969 & 0.0045 \\
\hline \multirow{4}{*}{ Sharpening } & Lena & 0.9969 & 0.0045 \\
\hline & Cameraman & 0.9213 & 0.1091 \\
\hline & Einstein & 0.9815 & 0.0273 \\
\hline & Zelda & 1 & 0 \\
\hline \multirow{4}{*}{$\begin{array}{l}\text { Crop (quarter from the center of the watermarked image and fill the missing } \\
\text { portion with } 0 \text { 's }\end{array}$} & Lena & 0.9143 & 0.1409 \\
\hline & Cameraman & 0.8573 & 0.2591 \\
\hline & Einstein & 0.9048 & 0.1591 \\
\hline & Zelda & 0.8822 & 0.2045 \\
\hline \multirow{4}{*}{$\begin{array}{l}\text { Crop (quarter from the center of the watermarked image and fill the missing } \\
\text { portion with host image }\end{array}$} & Lena & 1 & 0 \\
\hline & Cameraman & 0.9678 & 0.0455 \\
\hline & Einstein & 0.9969 & 0.0045 \\
\hline & Zelda & 0.9873 & 0.0182 \\
\hline \multirow{5}{*}{$\begin{array}{l}\text { Crop (quarter from the top right corner of the watermarked image and fill } \\
\text { the missing portion with 1's }\end{array}$} & Lena & 0.9937 & 0.0091 \\
\hline & Cameraman & 0.9937 & 0.0091 \\
\hline & Einstein & 0.9815 & \\
\hline & Zelda & 0.9242 & \\
\hline & & & 0.1227 \\
\hline \multirow{4}{*}{$\begin{array}{l}\text { Crop (quarter from the top right corner of the watermarked image and fill } \\
\text { the missing portion with host image }\end{array}$} & Lena & 1 & 0 \\
\hline & Cameraman & 1 & 0 \\
\hline & Einstein & 0.9969 & 0.0045 \\
\hline & Zelda & 1 & 0 \\
\hline \multirow{4}{*}{$\begin{array}{l}\text { Crop (quarter from the top left corner of the watermarked image and fill the } \\
\text { missing portion with 1's }\end{array}$} & Lena & 0.9906 & 0.0136 \\
\hline & Cameraman & 0.9815 & 0.0273 \\
\hline & Einstein & 0.9815 & 0.0273 \\
\hline & Zelda & 0.9584 & 0.0636 \\
\hline \multirow{4}{*}{$\begin{array}{l}\text { Crop (quarter from the top left corner of the watermarked image and fill the } \\
\text { missing portion with host image }\end{array}$} & Lena & 1 & 0 \\
\hline & Cameraman & 1 & 0 \\
\hline & Einstein & 0.9317 & 0.1091 \\
\hline & Zelda & 1 & 0 \\
\hline \multirow{4}{*}{$\begin{array}{l}\text { Crop (quarter from the bottom right corner of the watermarked image and } \\
\text { fill the missing portion with } 0 \text { 's }\end{array}$} & Lena & 0.9937 & 0.0091 \\
\hline & Cameraman & 0.8737 & 0.2227 \\
\hline & Einstein & 0.9969 & 0.0045 \\
\hline & Zelda & 0.9669 & 0.0500 \\
\hline \multirow{4}{*}{$\begin{array}{l}\text { Crop (quarter from the bottom right corner of the watermarked image and } \\
\text { fill the missing portion with host image }\end{array}$} & Lena & 1 & 0 \\
\hline & Cameraman & 0.9937 & 0.0091 \\
\hline & Einstein & & \\
\hline & Zelda & 0.9969 & 0.0045 \\
\hline \multirow{4}{*}{$\begin{array}{l}\text { Crop (quarter from the bottom left corner of the watermarked image and fill } \\
\text { the missing portion with 1's }\end{array}$} & Lena & 0.9119 & 0.1455 \\
\hline & Cameraman & 0.8933 & 0.1818 \\
\hline & Einstein & 0.9475 & 0.0818 \\
\hline & Zelda & 0.9786 & 0.0318 \\
\hline Crop & Lena & 0.9808 & 0.0273 \\
\hline
\end{tabular}


the missing portion with host image

\begin{tabular}{lll} 
Cameraman & 1 & 0 \\
Einstein & 1 & 0 \\
Zelda & 1 & 0 \\
Lena & 0.9292 & 0.1136 \\
Cameraman & 0.9529 & 0.0727 \\
Einstein & 0.9192 & 0.1318 \\
Zelda & 0.8613 & 0.2500 \\
\hline Lena & 0.9776 & 0.0318 \\
Cameraman & 0.9873 & 0.0182 \\
Einstein & 1 & 0 \\
Zelda & 0.8035 & 0.2545 \\
Lena & 1 & 0 \\
Cameraman & 0.9905 & 0.0136 \\
Einstein & 0.9513 & 0.0682 \\
Zelda & 1 & 0 \\
\hline Lena & 0.8751 & 0.1682 \\
Cameraman & 1 & 0 \\
Einstein & 0.9937 & 0.0091 \\
Zelda & 0.9968 & 0.0045 \\
\hline & &
\end{tabular}

\subsection{Comparison Results with Other Related Schemes}

In order to assess the effeciency of the proposed watermarking scheme, results reported in some related other existing schemes (W. Lin, S. Horng, T. Kao, P. Fan, C. Lee, Y. Pan, 2008), (B. Ma, Y. Wang, C. Li, Z. Zhang, D. Huang, 2012), (S. Wang, Y. Lin, 2004), (W. Lin, Y. Wang, S. Horng, T. Kao, Y. Pan, 2009), (Li, C., Zhang, Z., Wang, Y., Ma, B., \& Huang, D., 2015), (Yahya, A. N., Jalab, H. A., Wahid, A., \& Noor, R. M., 2015), (Jagadeesh, B., Kumar, P. R., \& Reddy, P. C, 2015) and (Agarwal, C., Mishra, A., \& Sharma, A, 2015) are compared with our results. The comparison includes several attacks as Median filter, Sharpening, Gaussian filter, Salt \& pepper, JPEG compression, Resizing, Rotation, and Cropping.

Our proposed watermarking scheme is compared to the other schemes based on the NC values reported in their results. Higher NC values indicate higher quality of the extracted watermark. As seen in Table 5, Table 6 and Table 7, most of NC results for our proposed watermarking scheme are higher than those reported by other scheme and that demonstrates the robusteness of the proposed watermarking scheme is far better and proves superiority over the other watermarking schemes.

Table 5. NC results comparison under different attacks using Lena image

\begin{tabular}{llllllllll}
\hline & \multicolumn{3}{c}{ Median filter Resize Crop Rotation } & \multicolumn{2}{c}{ Sharpening Gauss filter } \\
\cline { 2 - 7 } & $3 \times 3 \times 5 \times 5$ & 0.5 & $25 \%$ & -0.25 & 0.25 & & \\
\cline { 1 - 7 } (S. Wang... 2004) & - & - & - & - & 0.32 & 0.37 & 0.46 & 0.64 \\
(W. Lin... 2008) & 0.88 & 0.74 & 0.86 & 0.70 & 0.67 & 0.67 & 0.99 & 0.86 \\
(W. Lin... 2009) & 0.90 & 0.76 & 0.88 & 0.66 & 0.60 & 0.59 & 0.97 & 0.88 \\
(B. Ma .. 2012) & 0.94 & 0.42 & $\mathbf{0 . 9 8}$ & 0.87 & 0.38 & 0.35 & 0.21 & $\mathbf{1}$ \\
(Li, C... 2015) & 0.93 & 0.57 & 0.97 & 0.88 & 0.47 & 0.42 & 0.47 & 0.99 \\
Proposed scheme $\mathbf{1}$ & $\mathbf{0 . 9 9 0 6}$ & 0.8025 & $\mathbf{1}$ & $\mathbf{0 . 8 7 6 8}$ & $\mathbf{0 . 8 7 6 8}$ & $\mathbf{0 . 9 9 6 9}$ & 0.9906 \\
\hline
\end{tabular}

'-' means the attacks are not done.

The bold values indicate the best values comparing with the others.

Table 6. NC results comparison under JPEG compression attacks using Lena image

\begin{tabular}{llllllllll}
\hline \multicolumn{10}{c}{ JPEG Compression } \\
\cline { 2 - 9 } & 10 & 15 & 20 & 25 & 30 & 40 & 50 & 70 & 90 \\
\hline (S. Wang.. 2004) & - & - & - & - & 0.15 & 0.23 & 0.26 & 0.57 & $\mathbf{1}$ \\
(W. Lin... 2008) & 0.40 & 0.57 & 0.73 & 0.84 & 0.91 & 0.93 & 0.98 & $\mathbf{1}$ & $\mathbf{1}$ \\
(W. Lin... 2009) & 0.34 & 0.55 & 0.67 & 0.74 & 0.82 & 0.90 & 0.96 & 0.97 & 0.99 \\
\hline
\end{tabular}




\begin{tabular}{llllllllll}
\hline (B. Ma.. 2012) & 0.44 & 0.77 & 0.91 & 0.95 & 0.99 & $\mathbf{1}$ & $\mathbf{1}$ & $\mathbf{1}$ & $\mathbf{1}$ \\
(Li, C... 2015) & 0.63 & 0.85 & 0.92 & 0.95 & 0.97 & 0.99 & 0.99 & $\mathbf{1}$ & $\mathbf{1}$ \\
Proposed scheme & $\mathbf{0 . 9 5 7 1}$ & $\mathbf{0 . 9 7 8 6}$ & $\mathbf{0 . 9 9 3 7}$ & $\mathbf{1}$ & $\mathbf{1}$ & $\mathbf{1}$ & $\mathbf{1}$ & $\mathbf{1}$ & $\mathbf{1}$ \\
\hline
\end{tabular}

'-' means the attacks are not done.

The bold values indicate the best values comparing with the others.

Table 7. NC results comparison under different attacks using Lena image

\begin{tabular}{|c|c|c|c|c|c|c|c|c|c|c|}
\hline & \multicolumn{3}{|c|}{ Rotation } & \multicolumn{3}{|c|}{ JPEG Compression } & \multicolumn{2}{|c|}{ Cropping $25 \%$} & \multicolumn{2}{|c|}{ Med filter Salt \& pepper } \\
\hline & $10^{\circ}$ & $5^{\circ}$ & $45^{\circ}$ & 70 & 50 & 10 & upper sid & lower side & $3 \times 3$ & $1 \%$ \\
\hline (Yahya...2015) & - & 0.9251 & 0.8965 & 0.8451 & 0.7520 & 0.4395 & 0.6660 & 0.9023 & 0.9329 & - \\
\hline (Jagadeesh...2015) & 0.7549 & - & - & 0.8940 & - & - & 0.6456 & - & 0.7890 & 0.6450 \\
\hline (Agarwal... 2015) & - & - & - & 1 & 0.9931 & 0.4024 & 0.9734 & - & 0.9713 & - \\
\hline Proposed scheme & 0.8485 & 0.8573 & 0.8768 & 1 & 1 & 0.9571 & & 1 & 1 & 0.9906 \\
\hline
\end{tabular}

'-' means the attacks are not done.

The bold values indicate the best values comparing with the others.

\section{Conclusion}

In this paper, a robust watermarking scheme based on the original image content has been proposed and simulated. In this work, the steerable pyramid transform is used as an embedding domain due to its good spatial-frequency characteristics.

The experimental results of the proposed watermarking scheme have shown high perceptual quality of the watermarked image. In addition to the perceptual quality, our scheme achieves a good robustness against different intentional and non-intentional attacks.

\section{References}

Agarwal, C., Mishra, A., \& Sharma, A. (2015). A novel gray-scale image watermarking using hybrid Fuzzy-BPN architecture. Egyptian Informatics Journal, 16(1), 83-102. http://dx.doi.org/10.1016/j.eij.2015.01.002

Cheddad, A., Condell, J., Curran, K., \& Mc Kevitt, P. (2010). Digital image steganography: Survey and analysis of current methods. Signal Processing, 90(3), 727-752. http://dx.doi.org/10.1016/j.sigpro.2009.08.010

El Hossaini, A. E. A., El Aroussi, M., Jamali, K., Mbarki, S., \& Wahbi, M. (2014). Highly Robust Digital Image Watermarking Using Steerable Pyramid and Dual Encryption Technique. International Review on Computers and Software (IRECOS), 9(2), 255-265.

El Hossaini, A. E. A., El Aroussi, M., Jamali, K., Mbarki, S., \& Wahbi, M. (2016) A New Robust Blind Copyright Protection Scheme Based on Visual Cryptography and Steerable Pyramid. International Journal of Network Security, 18(2), 250-262.

Freeman, W. T., \& Adelson, E. H. (1991). The design and use of steerable filters. IEEE Transactions on Pattern Analysis \& Machine Intelligence, (9), 891-906.

Hossaini, E., El Arab, A., El Aroussi, M., Jamali, K., Mbarki, S., \& Wahbi, M. (2014). A New Robust Blind Watermarking Scheme Based on Steerable pyramid and DCT using Pearson product moment correlation. Journal of Computers, 9(10), 2315-2327. http://dx.doi.org/10.4304/jcp.9.10.2315-2327

Jagadeesh, B., Kumar, P. R., \& Reddy, P. C. (2015). Fuzzy Inference System Based Robust Digital Image Watermarking Technique Using Discrete Cosine Transform. Procedia Computer Science, 46, 1618-1625. http://dx.doi.org/10.1016/j.procs.2015.02.095

Jamali, K., El Aroussi, M., El Hossaini, A. E. A., Mbarki, S., \& Wahbi, M. (2014). A Robust Image Watermarking Scheme Using Steerable Pyramid Transform. International Review on Computers and Software (IRECOS), 9(10), 1750-1759. http://dx.doi.org/10.15866/irecos.v9i10.2238

Lee, C. H., \& Lee, Y. K. (1999). An adaptive digital image watermarking technique for copyright protection. Consumer Electronics. IEEE Transactions on, 45(4), 1005-1015. http://dx.doi.org/10.1109/30.809176

Li, C., Zhang, Z., Wang, Y., Ma, B., \& Huang, D. (2015). Dither modulation of significant amplitude difference for wavelet based robust watermarking. Neurocomputing. http://dx.doi.org/10.1016/j.neucom.2015.03.039 
Lin, W., Horng, S., Kao, T., Fan, P., Lee, C., \& Pan, Y. (2008). An efficient watermarking method based on significant difference of wavelet coefficient quantization. IEEE Transactions on Multimedia, 10(5), 746-757. http://dx.doi.org/10.1109/TMM.2008.922795

Lin, W., Wang, Y., Horng, S., Kao, T., \& Pan, Y. (2009). A blind watermarking method using maximum wavelet coefficient quantization. Expert Systems with Applications, 36(9), 11509-11516. http://dx.doi.org/10.1016/j.eswa.2009.03.060

Ma, B., Wang, Y., Li, C., Zhang, Z., \& Huang, D. (2012) A robust watermarking scheme based on dual quantization of wavelet significant difference, in: Proceedings of Pacific-Rim Conference on Multimedia (PCM). http://dx.doi.org/10.1007/978-3-642-34778-8_28

Wang, S., \& Lin, Y. (2004). Wavelet tree quantization for copyright protection watermarking. IEEE Transactions on Image Processing, 13(2), 154-165.

Yahya, A. N., Jalab, H. A., Wahid, A., \& Noor, R. M. (2015). Robust watermarking algorithm for digital images using discrete wavelet and probabilistic neural network. Journal of King Saud University-Computer and Information Sciences, 27(4), 393-401. http://dx.doi.org/10.1016/j.jksuci.2015.02.002

\section{Copyrights}

Copyright for this article is retained by the author(s), with first publication rights granted to the journal.

This is an open-access article distributed under the terms and conditions of the Creative Commons Attribution license (http://creativecommons.org/licenses/by/3.0/). 